\title{
Effect of Proanthocyanidin-rich Extracts from Pinus radiata Bark on Termite Feeding Deterrence ${ }^{1}$
}

\author{
Sung Phil Mun (iD) $2, \dagger$ Darrel D. Nicholas ${ }^{3}$
}

\begin{abstract}
Antioxidants are known to affect the feeding habits of termites and a good source is pine bark which contains high levels of antioxidants which can be extracted with neutral solvents. In this study procyanidins (PCs)-rich $\mathrm{MeOH}$-extract and a hot-water extract (HWE) were prepared from Pinus radiata bark. HWE was further separated into crude PCs (CPCs), polymeric PCs (PPCs), and low-molecular-weight PCs (LMWPCs) fractions. The $\mathrm{MeOH}$ extract and these fractions were examined for termite (Reticulitermes flavipes Kollar) antifeedant activities using a no-choice test. Catechin was used as a positive control. The LMWPCs was found to be the most effective deterrent to termite feeding. The comparative efficiency of the compounds tested were LMWPCs $>$ PPCs $>$ CPCs $>\mathrm{MeOH}$ extract, with the latter being more dependent on molecular weight than on its antioxidant activity.
\end{abstract}

Keywords: Pinus radiata bark, proantocyanidin-rich extracts, procyanidins (PCs), antioxidant activity, antifeedant activity, termite (Reticulitermes flavipes Kollar)

\section{INTRODUCTION}

Flavonoids are widely distributed in plants, and considered to be related to a plants resistance to insect and fungal attacks (Schultz et al., 1995; Ohmura et al., 2000; Boue and Raina, 2003; Binbuga et al., 2008; Little et al., 2010). Monomeric flavonoids having antioxidant activity, such as catechin and morin, are known to have good termite feeding deterrence and toxicity at treatment levels of $4 \%$ and $6 \%$ (Little et al., 2010). Although these flavonoids are reported to have antifeedant activity and toxicity against termites, an effort to commercialize may encounter a resistance because of their relatively high production cost. Procyanidins (PCs) composed of flavan 3-ol subunits (catechin unit) linked mainly through C4-C8 (or C6) bonds (Fig. 1) are a major component of Pinus radiata bark and have a high antioxidant activity ( $\mathrm{Ku}$ and Mun, 2007). PCs are easily extracted with a polar solvent such as

1 Date Received July 24, 2017, Date Accepted August 31, 2017

2 Department of Wood Science and Technology, Chonbuk National University, Jeonju, Jeonbuk, 54896, Republic of Korea

3 Department of Sustainable Bioproducts, Mississippi State University, Box 9820 Mississippi State, MS 39762, USA

${ }^{\dagger}$ Corresponding author: Sung Phil Mun (e-mail: msp@jbnu.ac.kr, ORCID: 0000-0002-3475-4965) 


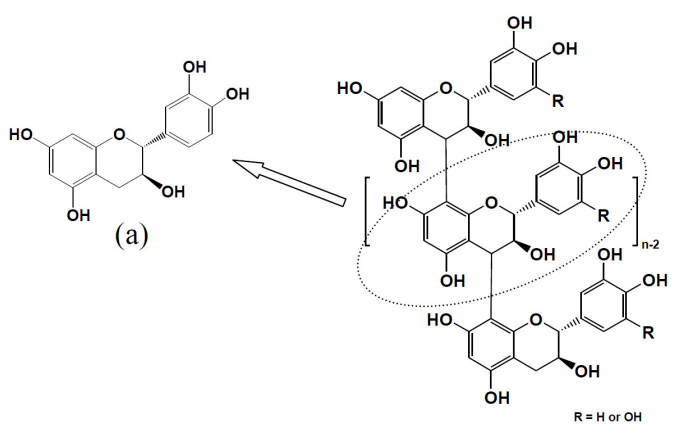

(b)

$\mathrm{R}=\mathrm{H}$ : Procyanidin, $\mathrm{R}=\mathrm{OH}$ : Prodelphinidin

Fig. 1. Chemical structures of catechin (a) and proanthocyanidins (b).

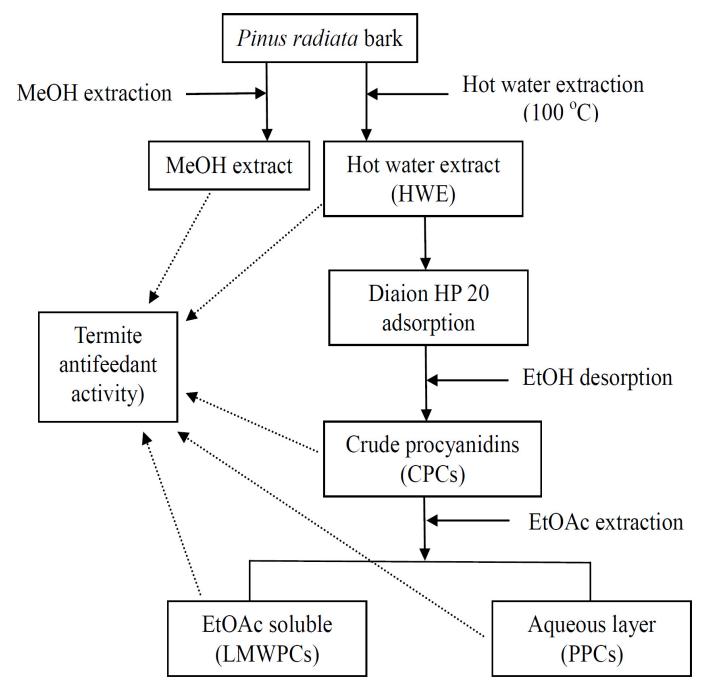

Fig. 2. Solvent extraction and separation scheme of different molecular weight's procyanidins from $P$. $r a-$ diata bark. PPCs: Polymeric procyanidins, LMWPCs: Low-molecular-weight procyanidins.

water (Ku et al., 2011), lower alcohols ( $\mathrm{Li}$ and Maplesden, 1998; Jerez et al., 2006), and weak-alkaline solutions (Mun and Kim, 2009), from $P$. radiata bark using a simple procedure which may lower the extraction cost. Presently, the product containing PCs as a major compound is selling in the market under the name of pine bark tannin for wood adhesion and the leather industry ( $\mathrm{Li}$ and Maplesden, 1998).

In this study, we investigated whether PCs-rich extracts such as $\mathrm{MeOH}$ extract, HWE, and PCs with high- and low-molecular weight distribution have antifeedant activities against termites in comparison to catechin in order to determine whether they have potential as environmentally-friendly termite control agents.

\section{MATERIALS and METHODS}

\subsection{Materials}

Southern yellow pine wafers measuring 25 $\mathrm{mm} \times 25 \mathrm{~mm} \times 6 \mathrm{~mm}(\mathrm{R} \times \mathrm{T} \times \mathrm{L})$ were cut from one sapwood lumber board for the termite test. The outer bark of $P$. radiata was provided from Unid Co. Ltd. in Kunsan, South Korea. The bark was dried in a convection oven at 60 $\pm 1{ }^{\circ} \mathrm{C}$ for 48 hours, ground using a Wiley mill equipped with a $1 \mathrm{~mm}$ screen. Methanol $(\mathrm{MeOH})$, ethyl acetate (EtOAc), and absolute ethanol (EtOH) were all HPLC grades purchased from Sigma-Aldrich Chemical (St. Louis, MO, USA) and used as extraction and/or desorption solvents to prepare various samples for the termite test from $P$. radiata bark. Diaion HP 20 was purchased from Mitsubishi Chemical (Tokyo, Japan). 


\subsection{Preparation of $\mathrm{MeOH}$ extract}

Fifty grams (oven dry weight: o.d.) of pine bark powder and $500 \mathrm{~m} \ell$ of $\mathrm{MeOH}$ were added to a 1-L Erlenmeyer flask. The flask was then placed in a hood at room temperature for 12 hours with occasional shaking. The slurry was filtered and the residue was washed with 200 $\mathrm{m} \ell$ of fresh $\mathrm{MeOH}$. The filtrate and washings were combined and evaporated to reduce the volume to about $150 \mathrm{~m} \ell$. This concentrated $\mathrm{MeOH}$ extract was then used in the termite antifeedant activity test. The residue was again washed with $600 \mathrm{~m} \ell$ of fresh $\mathrm{MeOH}$ and then dried at $130^{\circ} \mathrm{C}$ in a convection oven for 3 hours. $\mathrm{MeOH}$ extract yield was calculated from the decreased weight of the residue after $\mathrm{MeOH}$ extraction. The solid content in the $\mathrm{MeOH}$ extract was determined and used for calculating the treatment concentration of $\mathrm{MeOH}$ extract in the wood wafer.

\subsection{Preparation of HWE}

Sixty grams (o.d.) of the bark powder was extracted with $600 \mathrm{~m} \ell$ of de-ionized water (DI-water) in a 1-L beaker (liquor to bark ratio of 10). The beaker was then placed on a hot plate and allowed to boil for 1 hour with occasional stirring. The bark residue and HWE were separated by filtration using a $17 \mathrm{G} 3$ glass filter. The residue was washed with $600 \mathrm{~m} \ell$ DI-water, the filtrate and the washing were combined and used for preparation of crude procyanidins (CPCs), polymeric PCs (PPCs) and low-molec- ular-weight PCs (LMWPCs). HWE yields were determined in the same manner as the calculation for $\mathrm{MeOH}$ extract yield.

\subsection{Separation of CPCs, PPCs, and LMWCPS from HWE}

Diaion HP 20, commercial adsorbent, was used for the separation of CPCs, PPCs and LMWPCs from HWE as we previously reported (Mun and Kim, 2011; Mun, 2014). Diaion HP 20 (ca. $250 \mathrm{~m} \ell$ ), fully soaked and washed with EtOH and DI-water, and HWE were transferred to a 2-L Erlenmeyer flask. The adsorption was conducted at room temperature by agitating at $200 \mathrm{rpm}$ for 20 hours. The adsorbent was recovered by filtration and washed with $800 \mathrm{~m} \ell$ DI-water and then treated with EtOH to desorb the adsorbed materials onto the adsorbent. The solvent, EtOH, after desorption was removed by evaporation at $60^{\circ} \mathrm{C}$ and the resulting materials were then vacuum dried overnight at room temperature. We defined this fraction as crude procyanidins (CPCs). The CPCs were dispersed in DI-water and then further extracted with EtOAc. The EtOAc soluble fraction and the aqueous fraction were named LMWPCs and PPCs, respectively. Each fraction was evaporated to dryness at $60^{\circ} \mathrm{C}$.

\subsection{Antioxidant activity}

Antioxidant activities of samples prepared for this experiment were dissolved in $\mathrm{MeOH}$ and then measured by DPPH (1,1-diphenyl-2-pic- 
rylhydrazyl radical) free radical scavenging assay, with catechin being used as a positive control. In this method $10 \mathrm{mg}$ of each sample was dissolved in $10 \mathrm{~m} \ell$ of $\mathrm{MeOH}$. The sample solution was then diluted to $16.7 \mu \mathrm{g} / \mathrm{m} \ell$ with $\mathrm{MeOH}$. One milliliter of the dilute solution was mixed with $2 \mathrm{~m} \ell$ of $0.1 \mathrm{mM}$ DPPH $\mathrm{MeOH}$ solution in a test tube and reacted in a water bath at $25^{\circ} \mathrm{C}$ for 30 minutes. The absorbance was then measured at $518 \mathrm{~nm}$. The results were expressed as catechin equivalent antioxidant capacity.

DPPH free radical scavenging activity $(\%)=$ [1 - (absorbance of sample/absorbance of control) $] \times 100$

\subsection{Determination of molecular weight}

The samples prepared from this experiment were acetylated prior to conducting gel permeation chromatography (GPC) analysis. For acetylation, $20 \mathrm{mg}$ of each sample was placed in a $10 \mathrm{~m} \ell$ vial and dissolved in $2 \mathrm{~m} \ell$ of an acetic anhydride-pyridine $(1: 1, \mathrm{v} / \mathrm{v})$ mixture. The acetylation was performed for 48 hours at room temperature. For GPC analysis, $1 \mathrm{mg}$ of the acetylated sample was dissolved in $1 \mathrm{~m} \ell$ of tetrahydrofuran (THF) and was subsequently filtered using a syringe filter with a pore diameter of $0.45 \mu \mathrm{m}$. The filtrate was then subjected to GPC analysis. A Shimadzu LC-10AD pump unit equipped with a Spectra 100 variable detector (Spectra-Physics, USA) and an AM GEL column $(10 \mathrm{~mm} \times 30 \mathrm{~cm}$, American Polymer Standards, Co. Ltd., USA) was used for the experiments. The mobile phase was THF at a flow rate of $0.5 \mathrm{~m} \ell / \mathrm{min}$. The molecular weight (MW) calculation was based on a calibration curve obtained using monodisperse polystyrene standards and phenol. The effluent from the column was monitored at $280 \mathrm{~nm}$ for the sample and phenol, and at $254 \mathrm{~nm}$ for the polystyrene standards.

\subsection{Antifeedant activity}

All of the samples were dissolved in $\mathrm{MeOH}$ at a $6 \%(\mathrm{w} / \mathrm{w})$ level. The wood wafers of predetermined weights were soaked in each sample solution for 15 minutes and then placed in a vacuum oven at $30 \mathrm{mmHg}$ for 15 minutes at room temperature. Sample retentions were calculated from the weight differences before and after sample treatments. After treatment, the wafers were dried at $43^{\circ} \mathrm{C}$ for 2 hours and then placed in a conditioning chamber $\left(20^{\circ} \mathrm{C}, 12 \%\right.$ EMC) for 3 days until constant weights were obtained. $\mathrm{MeOH}$ treated wafers was used as controls. Reticulitermes flavipes Kollar termites were employed for the laboratory no-choice tests according to AWPA Standard E1 (AWPA, 2009). After 28 days exposure to the termites, the wood wafers were dried in the same manner as mentioned above to determine the weight loss, and then visually rated using the AWPA E1 scale of 10 to 0 . Five replications were conducted for each compound.

\subsection{Statistical analysis}

All data was reported as mean \pm standard de- 
Table 1. Yields of MeOH extract, HWE, CPCs, PPCs, and LMWPCs based on oven-dried bark weight

\begin{tabular}{ccccc}
\hline \multicolumn{5}{c}{ Yield $(\%$ of bark $)$} \\
\hline \hline $\mathrm{MeOH}$ extract & HWE & CPCs & PPCs & LMWPCs \\
\hline 32.9 & 28.9 & 23.9 & 18.1 & 5.8 \\
\hline
\end{tabular}

$\mathrm{HWE}=$ hot-water extract, $\mathrm{CPCs}=$ crude procyanidins, $\mathrm{PPCs}=$ polymeric procyanidins, LMWPCs $=$ low-molecular-weight procyanidins.

Table 2. $\overline{\mathrm{M}}_{\mathrm{W}}, \overline{\mathrm{M}}_{\mathrm{n}}$ and polydispersity $\left(\overline{\mathrm{M}}_{\mathrm{W}} / \overline{\mathrm{M}}_{\mathrm{n}}\right)$ of acetylated proantocyanidin-rich extracts from P. radiata bark

\begin{tabular}{cccccc}
\hline & MeOH extract & HWE & CPCs & PPCs & LMWPCs \\
\hline \hline$\overline{\mathrm{M}} \mathrm{w}$ & 6011 & 4281 & 3782 & 4120 & 925 \\
$\overline{\mathrm{M}} \mathrm{n}$ & 807 & 724 & 822 & 973 & 412 \\
$\overline{\mathrm{M}} \mathrm{w} / \overline{\mathrm{M}} \mathrm{n}$ & 7.4 & 5.9 & 4.6 & 4.2 & 2.2 \\
\hline
\end{tabular}

viation of five replicates. A statistical analysis was performed using the SAS 9.1 software package.

\section{RESULTS and DISCUSSION}

Fig. 2 shows the systematic preparation of $\mathrm{MeOH}$ extract, HWE, CPCs, PPCs and LMWPCs from $P$. radiata bark. The yields of $\mathrm{MeOH}$ extract and HWE were 33.6 and $28.9 \%$, respectively, as shown in Table 1 . The higher yield of $\mathrm{MeOH}$ extract for HWE is likely caused by the high solubility of procyanidins (PCs) in $\mathrm{MeOH}$ compared to that in hot water. Previous research reported that $\mathrm{MeOH}$ can easily extract PCs, as shown in Fig. 1, out of $P$. radiata bark (Li and Maplesden, 1998). HWE was soaked in a Diaion HP 20 adsorbent to separate CPCs, which yielded $82.7 \%$ of HWE, equating to a $23.9 \%$ yield based upon the bark weight. The PCs content determined by UV method (Park et al., 2011) in CPCs was 88\%, and they are known to contain a small amount of monomeric flavonoids and some unidentified hydrophobic compounds (Mun and Kim, 2011). CPCs can be separated to high- and low-molecular weight fractions by EtOAc extraction $(\mathrm{Ku}$ and Mun, 2007; Mun and Kim, 2011). PPCs and LMWCPs fractionated from EtOAc extraction of CPCs account for 18.1 and $5.5 \%$ of the bark weight, respectively (Table 1).

All of the samples prepared from $P$. radiata bark $(\mathrm{MeOH}$ extract, HWE, CPCs, PPCs and LMWPCs) were acetylated for the investigation of MW and the results are shown in Table 2. The $\mathrm{MeOH}$ extract showed the highest MW and polydispersity. This result indicates that a wide range of MW proanthocysnidins can be easily leached from the bark because of their high solubility in $\mathrm{MeOH}$ as mentioned above. The MWs of CPCs and PPCs, separated from HWE, were $3782 \mathrm{Da}$ and $4120 \mathrm{Da}$, respectively. The polydispersities of both samples (4.6 and 4.2, respectively) were similar to each other. In the case of LMWPCs, since the $\overline{\mathrm{M}} \mathrm{w}$ was $1000 \mathrm{Da}$ or less, it is likely that most of this fraction was composed of dimeric proan- 
Table 3. DPPH free radical scavenging activity, based on catechin equivalent antioxidant capacity (CEAC)

\begin{tabular}{cc}
\hline Sample & CEAC \\
\hline \hline Catechin & 1.00 \\
Pure PCs & 0.95 \\
CPCs & 1.03 \\
PPCs & 0.94 \\
LMWPCs & 0.96 \\
\hline
\end{tabular}

Pure PCs was prepared from $P$. radiata bark using a Sephadex LH 20. DPPH = 1,1-diphenyl-2-picrylhydrazyl radical.

thocyanoidins or smaller polyphenols.

All samples prepared except for HWE, which was found to have almost no activity against termites in a preliminary study, were evaluated in the termite antifeedant activity test.

As mentioned above, Little et al. (2010) reported that some monomeric flavonoids having antioxidant activity, such as morin and catechin, showed high termite feeding deterrence and toxicity. Table 3 shows the DPPH free radical scavenging activities of five samples prepared from $P$. radiata bark (pure PCs, CPCs, PPCs, and LMWPCs) and catechin as a positive control. In spite of the different MW of the aforementioned samples prepared from the bark, which are mainly composed of the catechin unit, they showed similar antioxidant activities to that of catechin. Catechin, flavan 3-ol, as shown in Fig. 1, is known as a potent antioxidant and has strong antifeedant activity against termites (Little et al., 2010). Therefore, we used catechin as a positive control and investigated the effectiveness of the $\mathrm{MeOH}$ extract and three different PCs-rich fractions having different MWs in the termite antifeedant activity test. As shown in Table 4, all samples prepared from the bark showed antifeedant activites against termites, but the effectiveness of them was somewhat lower than that of catechin, especially PPCs and $\mathrm{MeOH}$ extract which contains higher molecular weight PCs. The antifeedant activity increased in the order of $\mathrm{MeOH}$ extract, CPCs, PPCs, and LMWCPs, with LMWCPs exhibiting the highest activity. Although the samples used for the antifeedant activity test are mainly composed of catechin units linked through $\mathrm{C} 4-\mathrm{C} 8$ bonds and have similar antioxidant activities with catechin (Table 3), with the exception of LMWCPs they showed slightly lower antifeedant activities than catechin. The LMWCPs were the most effective deterrent to termite feeding. Ohmura et al. (1999; 2000) reported that larch wood showed a strong antitermite effect because it contains a high amount of the water soluble flavonoid, taxifolin. Some flavonoids isolated from Japanese larch wood, such as taxifolin, quercetin, aromadendrin, naringenin, along with some other monomeric flavonoids and aromatic acids were tested for the antifeedant effect against subtterranean termites. In a previous study, taxifolin, quercetin, and naringenin showed high antifeedant activities. Therefore, it was thought that the LMWCPs has a high antifeedant activity compared to catechin because it contains not only catechin, but also some monomeric flavonoids such as taxifolin and quercetine (Mun and $\mathrm{Ku}, 2006$; 2008). Consequently, the samples containing high amounts of PCs seemed to contribute positively to antifeedant 
Table 4. Average chemical retention, weight loss and ratings for wafers treated in the termite test

\begin{tabular}{cccc}
\hline Sample & Chemical retention, $\mathrm{kg} / \mathrm{m}^{3}$ & Weight loss, \% & Rating \\
\hline \hline MeOH & - & $29.7 \pm 3.6$ & $3.8 \pm 0.5$ \\
Catechin & $26.0 \pm 5.2$ & $16.1 \pm 2.1$ & $7.1 \pm 0.2$ \\
MeOH extract & $26.7 \pm 4.5$ & $22.6 \pm 5.1$ & $7.0 \pm 0.0$ \\
CPCs & $27.1 \pm 3.6$ & $20.1 \pm 3.3$ & $7.0 \pm 0.2$ \\
PPCs & $26.1 \pm 4.3$ & $18.0 \pm 1.5$ & $6.0 \pm 0.3$ \\
LMWPCs & $25.4 \pm 3.8$ & $13.8 \pm 1.6$ & 0.0 \\
\hline
\end{tabular}

Ratings are based on AWPA standard E1, where a 10 is no attack, 9 is a trace to $3 \%$ damage, down to 0 which is failure. Chemical treatment: $6 \%(\mathrm{w} / \mathrm{w})$.

activity, but the effectiveness was somewhat lower than that of catechin. Further study is needed to determine why higher molecular weight PCs showed lower activity against termites. The relationship, if any, between the antifeedant activity and antioxidant activity of the tested PCs-rich extracts and PCs having different molecular weight was not apparent in this study. However, this study showed that antifeedant activity was more dependent on the molecular weight of the PCs than on antioxidant activity.

\section{CONCLUSIONS}

The termite antifeedant activity of PCs-rich solvent extracts and PCs with different MWs prepared from $P$. radiata bark was investigated. CPCs, PPCs, and LMWPCs showed similar antioxidant activities to catechin, which is the basic unit of PCs used as a positive control. However, antifeedant activity of the PCs against termites was somewhat lower than that of catechin, especially for higher molecular weight compounds. The relationship between antifeedant activity and antioxidant activity of the tested PCs-rich extracts and PCs having different
MW was not clear, but the antifeedant activity of PCs appears to be more dependent on their molecular weight than on their antioxidant activity.

\section{ACKNOWLEDGEMENTS}

The authors would like to thank Dr. T. Schultz, a former Mississippi State University professor who participated in this study. We also would like to thank Mrs Amy Rowlen, a research associate at Mississippi State University, for her support.

\section{REFERENCES}

AWPA 2009. American Wood Protection Association Standard E1. Standard method for laboratory evaluation to determine resistance to subterranean termites.

Boue, S., Raina, A.K. 2003. Effects of plant flavonoids on fecundity, survival, and feeding of the Formosan subterranean termite. Journal of Chemical Ecology 29: 2575 2584.

Binbuga, N., Ruhs, C., Hasty, J.K., Henry, W.P., Schultz, T.P. 2008. Developing environmentally benign and effective organic wood preservatives by understanding the biocidal and non-biocidal properties of extractives in naturally durable heartwood. Holzforschung 62: 264 269. 
Jerez, Pinelo, M., Sineiro, M.J., Nunez, M.J. 2006. Influence of extraction conditions on phenolic yields from pine bark: assessment of procyanidins polymerization degree by thiolysis. Food Chemistry 94: 406 414.

Ku, C.S., Jang, J.P., Mun, S.P. 2007. Exploitation of polyphenol-rich pine barks for potent antioxidant activity. Journal of Wood Science 53: 524 528 .

Ku, C.S., Mun, S.P. 2007. Characterization of proanthocyanidin in hot water extract isolated from Pinus radiata bark. Wood Science and Technology 41: 235 247.

$\mathrm{Ku}$, C.S., Mun, S.P. 2008. Antioxidant properties of monomeric, oligomeric and polymeric fractions in hot water extract from Pinus radiata bark. Wood Science and Technology 42: 47 60 .

$\mathrm{Ku}$, C.S., Mun, S.P., Jang, J.P. 2011. Effects of water extraction temperatures on the yield, molecular weight, and antioxidant activity of proanthocyanidins extracted from Pinus radiata bark. Forest Products Journal 61(4): 321 325.

Li, J., Maplesden, F. 1998. Commercial production of tannins from radiata pine bark for wood adhesives. IPENZ Transactions 25(1): 46 52 .

Little, N.S., Schultz, T.P., Nicholas, D.D. 2010. Termite-resistant heartwood. Effect of antioxidants on termite feeding deterrence and mortality. Holzforschung 64: 395 398.

Mun, S.P., Ku, C.S. 2006. Characterization of low molecular weight polyphenols from pine (Pinus radiata) bark. Food Science and Biotechnology 15(3): $424 \sim 430$.

Mun, S.P., Kim, S.J. Extraction of proanthocyanidin-rich extract from Pinus radiata bark under mild alkaline condition. In Abstract of the 59th Annual Meeting of the Japan Wood Research Society, March 14 - 17, 2009, Nagano Japan;
Japan Wood Research Society, Tokyo Japan. p. 72 .

Mun, S.P. 2014. Efficacy and reusability of commercial adsorbent for isolation of proanthocyanidins from hot water extract of Pinus radiata bark. Journal of The Korean Wood Science \& Technology 42(2): 207 213 .

Mun, S. P., Kim, B. H. Separation and molecular fractionation of proanthocyanidins from hot water extract of Pinus radiata bark. In Abstracts of the 61th Annual Meeting of the Japan Wood Research Society, March 17 - 20, 2011, Kyoto Japan; Japan Wood Research Society, Tokyo Japan. p. 67.

Ohmura, W., Doi, S., Aoyama, M., Ohara, S. 1999. Components of steamed and non-steamed Japanese larch (Larix leptolepis (Sieb. Et Zucc.) Gord.) heartwood affecting the feeding behavior of the subterranean termite, Coptotermes formosanus Shiraki (Isoptera: Rhinotermitidae). Holzforschung 53(6): 569 574.

Ohmura, W., Doi, S., Aoyama, M., Ohara, S. 2000. Antifeedant activity of flavonoids and related compounds against the subterranean termite Coptotermes formosanus Shiraki. Journal of Wood Science 46: 149 153.

Park, I.J., Cha, S.Y., Kang, M., So, Y.S., Go, H.G., Mun, S.P., Ryu, K.S., Jang, H.K. 2011. Effect of proanthocyanidin-rich extract from Pinus radiata bark on immune responses of specific-pathogen-free White Leghorn chickens. Poultry Science 90: 977〜982.

Schultz, T.P., Harms, W.B., Fisher, T.H., McMurtrey, K.D., Minn, J., Nicholas, D.D. 1995. Durability of angiosperm heartwood: The importance of extractives. Holzforschung 49: $29 \sim 34$. 